\title{
NUCLEAR FUNCTION AND RELEASE OF |L-33
}

\author{
Dr. Mousa Komai-koma
}

Department of Hematology \& Immunology,

Faculty of Medicine,

Umm Al Qura University,

Makkah, Saudi Arabia.

P.O. Box 50431

\section{Correspondence Address:}

Dr. Mousa Komai-koma

Department of Hematology \&

Immunology,

Faculty of Medicine,

Umm Al Qura University,

Makkah, Saudi Arabia.

P.O. Box 50431

mkkoma@uqu.ed.sa

mousakomaikoma@gmail.com

Article received on:

13/02/2014

Accepted for Publication:

01/04/2014

Received after proof reading

$28 / 05 / 2014$

\section{INTRODUCTION}

IL-33 was recognized as a novel IL-1 family member including IL- $1 \alpha, \quad \mathrm{IL}-1 \beta$ and $\mathrm{IL}-18^{1-3}$. Interleukin-33 is a pleiotropic cytokine that can initiate different immune response based on signals via a heterogeneous receptors consisting of ST2 and IL-1R accessory protein and mainly activate NF-kB and MAPKs ${ }^{1-3}$. IL-33 mRNA is broadly expressed in a variety of nonhematopoietic cells including fibroblasts, adipocytes, smooth muscle, endothelial, and bronchial epithelial cells and in restricted populations of inflammatory cells such as macrophages and dendritic cells ${ }^{2,4}$. IL-33 releases when cells undergo necrosis but not in apoptosis, such as in infections ${ }^{2,5,6}$. IL-33 binds strongly to its receptor, ST2 that is expressed on all innate immune cells. It also was initially identified as an inducer of Th2 responses in naive mice ${ }^{1,3,7-9}$, and subsequently with specific Th2 responses in allergic and parasitic diseases ${ }^{2,410}$. ST2 exists in two different splice variants leading to the synthesis of ST2L, a transmembrane receptor that confers IL-33's biologic effects, and sST2, a soluble molecule that likely serves as a decoy receptor for IL-33 ${ }^{11}$. The biologic relevance of both ST2 and IL-33 isoforms is unknown in human disease. Signaling via ST2 can induce both antigen-dependent and antigen-independent immune responses by directly activating a wide range of innate immune cells including eosinophils, macrophages, mast cells, B1 cells or Thelper type 2 (Th2) and IL-5+ Th cells in vitro and in vivo ${ }^{7,9,12,13}$. In addition, IL-33 can also promote Th1 and/or Th17 type responses in proinflammatory disorders in mice, by as yet undefined mechanisms $\left({ }^{14,15}\right.$ and unpublished data). The IL-33/ST2 axis seems to play a vital role in several chronic inflammatory disorders, including asthma, rheumatoid arthritis, and 
anaphylactic shock $\mathrm{k}^{4,7,10,13,16}$ and play a pathogenic role in inflammatory bowel disease ${ }^{17}$. Interleukin33 and ST2 expression is increased in inflamed colonic mucosa and in the serum of patients with inflammatory bowel disease ${ }^{15,17}$. Experimental IL33 gene-deletion impairs pathogenesis of colitis, ${ }^{17}$ although the mechanisms by which the IL-33/ST2 system exacerbates colitis are unresolved.

It was shown that the IL-33 exerts specific effects in the gut, because mice treated with recombinant IL33 induced epithelial hyperplasia and eosinophil/neutrophil infiltration in the colonic mucosa ${ }^{2}$. Similar to IL-1 $\alpha$, IL-33 may possess dual roles, functioning as an intracellular nuclear factor and a proinflammatory cytokine ${ }^{18}$; in fact, IL-33 has been described as a chromatin-associated transcriptional regulating factor ${ }^{19}$. Recently, our collaborators have shown that the IL-33 can protect mice against atherosclerosis ${ }^{20}$ and sepsis and the IL-33 levels are very high in the sepsis patients $^{21}$. Moreover, IL-33 can potentially induce adaptive immune responses to intestinal nematodes and helps in the expulsion of the worms from the intestine ${ }^{22}$.

\section{IL33 proteolytic processing}

Due to IL-33's homology to other IL-1 family cytokines it was initially thought that IL-33 would be expressed as an inactive pro-enzyme requiring proteolytic maturation by either caspase I, caspase 8 or calpain ${ }^{2,23-25}$. Indeed Schmitz and colleagues showed that IL-33, like IL-1 $\beta$ and IL-18 could be cleaved by caspase I in vitro. However, the fact that IL-33 orthologs from various other species lack consensus, caspase I sites cast doubt on the requirement of caspase I cleavage for bio-activity. Subsequently IL-33 like IL-1 $\alpha$ has been shown to be biologically active in its full-length form, indeed cleavage by caspase 1 results in the production of a truncated form that is unable to signal $^{5,6}$. Further controversy over this issue has arisen from the recent report that recombinant fulllength IL-33 (fl-IL-33) fails to signal via ST2 ${ }^{26}$. However, since the authors did not activate the flIL-33 by proteolytic cleavage, demonstrating activity, it is unclear if this finding reflects the biology of fl-IL-33. IL-33 is also cleaved by the apoptosis associated proteases, caspase- 3 and 7 which effectively destroy IL-33's ability to signal, however, interestingly the $\mathrm{N}$-terminal fragment containing the transcription factor activity still localizes to the nucleus and potentially may still fulfill some gene regulatory role there ${ }^{27}$. Intriguingly circulating IL-33 from Crohn's patients is entirely made up of a shortened form (approx. $20 \mathrm{kDa})^{28}$. The authors suggested that this 'cleaved' form was the result of proteolytic cleavage, possibly by one of the apoptosisassociated caspases 3 and 7 . Given these findings it is curious that the truncated 'mature' form originally described by Schmitz and colleagues has been used in all instances were the role of IL33 has been assessed in vivo, a molecule that in all likelihood does not exist in nature. It would be interesting to compare the in vivo activities of the truncated recombinant IL-33 with that of the fulllength protein.

\section{IL-33 cellular source}

\section{1) IL-33 as a body barrier alarmin}

First described as being a nuclear factor expressed specifically in high endothelial venules, it has subsequently been found to be broadly and constitutively expressed in cells of many blood barriers $^{30}$. This has lead to the suggestion IL-33 acts as an alarmin, its passive release from damaged or dying cells signals to cell of the innate immune system alerting them to the presence of potentially harmful pathogens ${ }^{29,30}$. In this regard IL33 is thought to act in a manner similar to the archetypal alarmin, High mobility group box 1(HMGB1), both are released from dying cells and both are nuclear factors, HMGB1 having well characterized roles in gene regulation, chromatin remolding, and DNA replication and repair ${ }^{31}$. HMGB1 when released from necrotic cells acts as a potent pro-inflammatory cytokine signaling via the RAGE receptor ${ }^{32}$. However, HMGB1 signaling via RAGE is only able to illicit pro-inflammatory responses when bound to other co-stimulatory molecules, including LPS, ssDNA, IL1B and nucleosomes which induce potent inflammatory responses via TLR4, TLR9, IL1 receptor and TLR2, respectively $^{33}$. Like HMGB1 IL-33 has also been shown to interact with heterochromatin and mitotic 
chromatin $^{34}$. It is unclear in what form IL-33 is released from necrotic cells; it is possible that IL-33 spills from dying cells complexed with other nuclear components. This might as is case with HMGB1 dramatically influence the manner and/or extent to which inflammatory cells respond to IL33. A thorough characterization of the components of any released IL-33 complexes and an assessment of any potential co-stimulatory factors that may be present would be invaluable. Although, IL-33's role as an alarmin has gained wide acceptance, it is however, unclear why barrier cells have exploited IL-33 when the same cells also express HMGB1.

Like HMGB1, IL-33 is released from necrotic cells whilst being actively retained complexed with the chromatin of apoptotic cells ${ }^{32}$. How IL-33 achieves this is yet to be described. The situation for HMGB1 is in contrast better defined; apoptosis induced hyper-acetylation of chromatin increases its affinity for HMGB1 preventing it from being released, whilst in necrotic cells HMGB1 is modified by poly (ADP) ribosylation allowing it to translocate from the nucleus to the cytoplasm ${ }^{31}$. To date no post-translation modifications of IL-33 have been described.

The biology of IL-33 shares several common features with IL-1 1 : they are both reported to be active in their full-length forms, both are released during necrosis and both have dual roles as proinflammatory cytokines, and nuclear factors. IL-33 being constitutively nuclear in most cell types, IL$1 \alpha$ translocating to the nucleus upon stimulation with LPS ${ }^{18,34}$. However, IL-33 is likely to act as a transcriptional repressor, whilst IL-1 $\alpha$ has been shown to have transcriptional activator activities ${ }^{18}$.

\section{IL-33 cellular source}

(2) Induced expression and secretion of IL-33 from inflammatory cells

IL-33 expression and active secretion have been observed in a variety of inflammatory cell types including: monocytes, macrophages, astrocytes and synovial fibroblasts, in response to stimulation with a range of pro-inflammatory signals, LPS, IL-1 $\beta$, and TNF- $\alpha^{2,14,35-37}$. Like IL-1 $\beta$, IL-18 and HMGB1, IL33 lacks a signal peptide thus cannot be mobilized via the classical ER-Golgi exocytotic pathway. Some reports present data suggesting that IL-33 secretion may involve similar processes to those thought to occur for IL-1 $\beta$ and IL-18. The release of $\mathrm{IL}-1 \beta$ and IL-18 requires two signals, an initial signal in the form of PAMP, such as LPS, stimulates gene expression, subsequent exposure to ATP activates the nucleotide gated ion channel P2X7 resulting in an influx of $\mathrm{K}+$ which along with LPS and a drop in cytosolic $\mathrm{Ca}_{2}$ levels activates the inflammasome resulting in the activation of caspase-I their maturation into bioactive cytokines and ultimately their release ${ }^{38,39}$. As has been discussed previously, IL-33 is unlikely to be a substrate of capase-1 but may still require caspase 1 activity to be secreted as is the case for $\mathrm{HMGB} 1^{40}$. In line with this theory astrocytes have been observed to release significant amounts of IL-33 when treated with LPS and ATP ${ }^{35}$. LPS induces IL-33 expression that localizes to the nucleus, subsequent incubation with ATP results in its mobilization from the nucleus to the cytoplasm at which time extracellular IL-33 can be detected. However, this does not seem broadly true for all inflammatory cell types. Nile and collegues showed that IL-33 expression could be induced by PAMP stimulation in primary human monocytes and a monocyte cell line ${ }^{37}$. However they failed to detect the release of IL33 even upon incubation with ATP, conditions which elicited the secretion of both IL1 $\beta$ and IL18. Moreover it has been shown that IL33 release is unimpaired in caspase- 1 deficient monocytes ${ }^{36}$.

IL-33's presence within the nucleus represents a significant barrier to IL-33 secretion, unlike IL-1 $\beta$ and IL18; IL-33 must negotiate passage through both nuclear and cell membranes. HMGB1 which must undertake a similar passage accomplishes this by encoding a nuclear export sequence (NES) and via a variety of post-translational modifications including: acetylation, phosphorylation, methylation and poly (ADP) ribosylation all of which serve to redirect HMGB1 out of nucleus and /or prevent it from re-entering it ${ }^{31}$. The mechanism of IL-33's egress from the nucleus is unknown, 
IL33 does not contain a NES and to date no posttranslational modifications have been described. A characterization of IL-33 post-translational modifications would provide invaluable insight into how IL-33 is mobilized from the nucleus and ultimately secreted. Although IL-33 is localized within the nucleus in the majority of cells that express it, a review of published IL-33 immunostaining reveals a few notable exceptions. IL-33 is localized within the cytoplasm of airway smooth muscle cell bundles from asthmatic patients, cells that also secrete small but significant amounts of IL-33 ${ }^{41}$. Cytoplasmic IL-33 staining is also evident in intestinal epithelial cells taken from patients suffering from ulcerative colitis and Crohn's disease, serum from the same patients show high serum levels of IL-33 $(2 \mathrm{ug} / \mathrm{ml})$, almost all of which is a $(20 \mathrm{kDa})$ short form of IL33 ${ }^{28}$. Again the mechanism by which IL-33 accumulates in the cytoplasm of these cells is unknown; one possibility is that IL-33 is prevented from entering the nucleus due to loss of NLS, either as a result of proteolytic cleavage or by being expressed as a splice variant that does not encode it. Both of which would result in a shortened form of IL-33. A splice isoform of human IL-33 does exist which lacks the first two coding exons, the resultant protein is predicted to be $16 \mathrm{kDa}$ and would as it lacks the NLS be localize primarily within the cytoplasm (ENST00000417746). A recent publication has identified a novel splice variant of human IL-33 that omits exon3. The resulting protein lacks the hypothetical caspase I site predicted by Schmitz et al., however, the resulting protein retains the homeobox-like domain and residues reported to be essential for nuclear localization $^{26}$.

Interestingly, IL-33 and ST2L are co-expressed in many cell types: including Synovial fibroblasts, astrocytes and monocytes ${ }^{2,14,35,37,42}$. This raises the possibility that IL-33 could act in an autocrine manner signaling directly via ST2L expressed on the same cell. IL-1 $\beta$ and IL-18 have previously been shown capable of signaling via co-expressed receptors ${ }^{43,44}$.

\section{Nuclear Function of IL33}

Undoubtedly the greatest mystery of II33 biology is the role it plays within the nucleus. IL-33's Nterminus contains a domain that has been predicted to be a member of the homeobox-like helix-turn-helix (HTH) family of DNA binding proteins, the founding member of which is engrailed protein from Drosophila ${ }^{45}$. The $\mathrm{N}$ terminal HTH has subsequently been shown to be required for IL-33's association with heterochromatin, and essential for its transcriptional repressor activity ${ }^{19}$. The same region contains residues, which are required for IL-33's nuclear localisation. Roussel and colleagues found that IL33 could negatively regulate gene expression by promoting interactions between histones $\mathrm{H} 2 \mathrm{~A}$ $\mathrm{H} 2 \mathrm{~B}$ inducing chromatin compaction ${ }^{34}$. To date the identities of IL-33 target genes remains unknown; it is discovery of these genes that represents the next significant milestone towards a fuller understanding of the biology of IL-33.

Copyright @ 01 Apr, 2014.

\section{REFERENCES}

1. Ali, S. et al. IL-1 receptor accessory protein is essential for IL-33-induced activation of $T$ lymphocytes and mast cells. Proceedings of the National Academy of Sciences 104, 18660-18665 (2007).

2. Schmitz, J. et al. IL-33, an interleukin-1-like cytokine that signals via the IL-1 receptor-related protein ST2 and induces T helper type 2associated cytokines. Immunity 23, 479-490 (2005).

3. Chackerian, A. A. et al. IL-1 receptor accessory protein and ST2 comprise the IL-33 receptor complex. J. Immunol. 179, 2551-2555 (2007).

4. Liew, F. Y., Pitman, N. I. \& Mclnnes, I. B. Diseaseassociated functions of IL-33: the new kid in the IL-1 family. Nat. Rev. Immunol. 10, 103-110 (2010).

5. Cayrol, C. \& Girard, J.-P. The IL-1-like cytokine IL33 is inactivated after maturation by caspase-1. Proceedings of the National Academy of Sciences 106, 9021-9026 (2009).

6. Lüthi, A. U. et al. Suppression of interleukin-33 bioactivity through proteolysis by apoptotic caspases. Immunity 31, 84-98 (2009).

7. Verri, W. A. et al. IL-33 induces neutrophil 
migration in rheumatoid arthritis and is a target of anti-TNF therapy. Ann. Rheum. Dis. 69, 1697-1703 (2010).

8. Xu, D., Chan, W. L., Leung, B. P. \& Huang, F. Selective expression of a stable cell surface molecule on type 2 but not type 1 helper T cells. The Journal of ... (1998).

9. Koma, M. K., Xu, D. \& Li, Y. IL-33 is a chemoattractant for human Th2 cells. European journal of ... (2007).

10. Kurowska-Stolarska, M. et al. IL-33 induces antigen-specific IL-5+ T cells and promotes allergic-induced airway inflammation independent of IL-4. J. Immunol. 181, 4780-4790 (2008).

11. Trajkovic, V., Sweet, M. J. \& Xu, D. T1/ST2-an IL-1 receptor-like modulator of immune responses. Cytokine \& growth factor reviews (2004).

12. Komai-Koma, M. et al. IL-33 activates B1 cells and exacerbates contact sensitivity. J. Immunol. 186, 2584-2591 (2011).

13. Komai-Koma, M. et al. Interleukin-33 amplifies IgE synthesis and triggers mast cell degranulation via interleukin-4 in naïve mice. Allergy 67, 1118-1126 (2012).

14. $\mathrm{Xu}, \mathrm{D}$. et al. IL-33 exacerbates antigen-induced arthritis by activating mast cells. Proceedings of the National Academy of Sciences 105, 10913-10918 (2008).

15. Pushparaj, P. N., Li, D. \& Koma, M. K. Interleukin IL33 exacerbates acute colitis via Interleukin IL-4 in mice Immunology 140, 70-7 (2013).

16. Xu, D., Jiang, H. R., Li, Y. \& Pushparaj, P. N. IL-33 exacerbates autoantibody-induced arthritis. The Journal of Immunology 184, 2620-6 (2010).

17. Latiano, A. et al. Associations between genetic polymorphisms in IL-33, IL1R1 and risk for inflammatory bowel disease. PLoS ONE 8, e62144 (2013).

18. Werman, A. et al. The precursor form of IL-1alpha is an intracrine proinflammatory activator $0 \quad f$ transcription. Proc. Natl. Acad. Sci. U.S.A. 101, 2434-2439 (2004).

19. Carriere, V. et al. IL-33, the IL-1-like cytokine ligand for ST2 receptor, is a chromatin-associated nuclear factor in vivo. Proc. Natl. Acad. Sci. U.S.A. 104, 282-287 (2007).
20. Miller, A. M., Xu, D., Asquith, D. L., Denby, L. \& Li, Y. IL-33 reduces the development of atherosclerosis. The Journal of experimental medicine 205, 339-46 (2008).

21. Alves-Filho, J. C., Sônego, F., Souto, F. O. \& Freitas, A. Interleukin-33 attenuates sepsis by enhancing neutrophil influx to the site of infection. Nature medicine (2010).

22. Humphreys, N. E., Xu, D. \& Hepworth, M. R. IL-33, a potent inducer of adaptive immunity to intestinal nematodes. The Journal of ... (2008).

23. Dinarello, C. A. Biologic basis for interleukin-1 in disease. Blood 87, 2095-2147 (1996).

24. Robertson, S. E. et al. Expression and alternative processing of IL-18 in human neutrophils. Eur. J. Immunol. 36, 722-731 (2006).

25. Maelfait, J. et al. Stimulation of Toll-like receptor 3 and 4 induces interleukin-1beta maturation by caspase-8. Journal of Experimental Medicine 205, 1967-1973 (2008).

26. Hong, J. et al. Identification of constitutively active interleukin 33 (IL-33) splice variant. J. Biol. Chem. 286, 20078-20086 (2011).

27. Ali, S., Nguyen, D. Q., Falk, W. \& Martin, M. U. Caspase 3 inactivates biologically active full length interleukin-33 as a classical cytokine but does not prohibit nuclear translocation. Biochemical and biophysical ... (2010).

28. Pastorelli, L., Garg, R. R. \& Hoang, S. B. Epithelialderived IL-33 and its receptor ST2 are dysregulated in ulcerative colitis and in experimental Th1/Th2 driven enteritis. in (2010).

29. Gadina, M. \& Jefferies, C. A. IL-33: a sheep in wolf's clothing? Sci. STKE 2007, pe31 (2007).

30. Moussion, C., Ortega, N. \& Girard, J. P. The IL-1-like cytokine IL-33 is constitutively expressed in the nucleus of endothelial cells and epithelial cells in vivo: a novel 'alarmin'? PLOS ONE (2008).

31. Stros, M. HMGB proteins: interactions with DNA and chromatin. Biochim. Biophys. Acta 1799, 101-113 (2010).

32. Scaffidi, P., Misteli, T. \& Bianchi, M. E. Release of chromatin protein HMGB1 by necrotic cells triggers inflammation. Nature 418, 191-195 (2002).

33. Bianchi, M. E. \& Manfredi, A. A. High-mobility group 
box 1 (HMGB1) protein at the crossroads between innate and adaptive immunity. Immunological reviews (2007).

34. Roussel, L., Erard, M., Cayrol, C. \& Girard, J.-P. Molecular mimicry between IL-33 and KSHV for attachment to chromatin through the H2A-H2B acidic pocket. EMBO Rep. 9, 1006-1012 (2008).

35. Hudson, C. A. et al. Induction of IL-33 expression and activity in central nervous system glia. J. Leukoc. Biol. 84, 631-643 (2008).

36. Ohno, T. et al. Caspase-1, caspase-8, and calpain are dispensable for IL-33 release by macrophages. J. Immunol. 183, 7890-7897 (2009).

37. Nile, C. J., Barksby, E., Jitprasertwong, P., Preshaw, P. M. \& Taylor, J. J. Expression and regulation of interleukin-33 in human monocytes. Immunology $130,172-180$ (2010).

38. Ferrari, D., Pizzirani, C. \& Adinolfi, E. The P2X7 receptor: a key player in IL-1 processing and release. The Journal of 176, 3877-83 (2006).

39. Lamkanfi, M. \& Dixit, V. M. Inflammasomes: guardians of cytosolic sanctity. Immunological reviews (2009).
40. Lamkanfi, M. et al. Inflammasome-dependent release of the alarmin HMGB1 in endotoxemia. J. Immunol. 185, 4385-4392 (2010).

41. Préfontaine, D. et al. Increased expression of IL-33 in severe asthma: evidence of expression by airway smooth muscle cells. J. Immunol. 183, 5094-5103 (2009).

42. Mun, S. $\mathrm{H}$. et al. Interleukin-33 stimulates formation of functional osteoclasts from human CD14(+) monocytes. Cell. Mol. Life Sci. 67, 3883-3892 (2010).

43. Fratelli, M. et al. Autocrine interleukin-1 beta regulates both proliferation and apoptosis in EL4-6.1 thymoma cells. Blood 85, 3532-3537 (1995).

44. Fortin, C. F., Ear, T. \& McDonald, P. P. Autocrine role of endogenous interleukin-18 on inflammatory cytokine generation by human neutrophils. FASEB J. 23, 194-203 (2009).

45. Baekkevold, E. S. et al. Molecular characterization of NF-HEV, a nuclear factor preferentially expressed in human high endothelial venules. Am. J. Pathol. 163, 69-79 (2003).

\section{6 \\ friend to all is a friend to none.}

\section{Aristotle}

